\title{
Revisiting TRPC1 and TRPC6 mechanosensitivity
}

Philip Gottlieb $^{1^{*}}$, Joost Folgering ${ }^{2^{*}}$, Rosario Maroto ${ }^{3^{*}}$, Albert Raso ${ }^{4}$, Thomas G. Wood $^{5}$, Alex Kurosky ${ }^{5}$, Charles Bowman', Delphine Bichet ${ }^{2}$, Amanda Patel' Frederick Sachs ${ }^{1}$, Boris Martinac ${ }^{6}$, Owen P. Hamill ${ }^{3 *}$ and Eric Honoré ${ }^{2 *}$

${ }^{1}$ Single Molecule Biophysics, 301 Cary Hall, SUNY, Buffalo, NY 14214 USA

2IPMC-CNRS, UMR 6097, Université de Nice-Sophia Antipolis, 06560 Valbonne, France

${ }^{3}$ Department of Neuroscience \& Cell Biology, UTMB, Galveston, TX 77555, USA

${ }^{4}$ School of Medicine and Pharmacology, University of Western Australia,

Crawley, WA 6009, Australia

${ }^{5}$ Department of Biochemistry and Molecular Biology, UTMB, Galveston, TX 77555, USA

${ }^{6}$ School of Biomedical Sciences, University of Queensland, St Lucia, Brisbane, QLD 4072, Australia

${ }^{*}$ co-first authors : PG, JF and RM

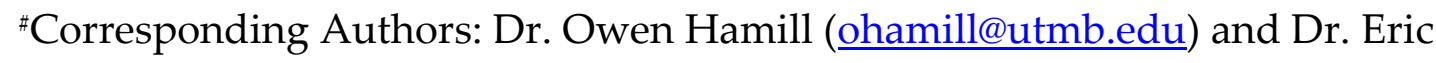
Honoré (honore@ipmc.cnrs.fr) 


\begin{abstract}
This article addresses whether TRPC1 or TRPC6 are essential components of a mammalian stretch-activated mechano-sensitive $\mathrm{Ca}^{2+}$ permeable cation channel (MscCa). We have transiently expressed TRPC1 and TRPC6 in COS or $\mathrm{CHO}$ cells and monitored the activity of the stretch-activated channels using a fast pressure clamp system. Although both TRPC1 and TRPC6 are highly expressed at the protein level, the amplitude of the mechano-sensitive current is not significantly altered by overexpression of these subunits. In conclusion, although several TRPC channel members, including TRPC1 and TRPC6, have been recently proposed to form $\mathrm{MscCa}$ in vertebrate cells, the functional expression of these TRPC subunits in heterologous systems remains problematic.
\end{abstract}

\title{
Introduction
}

$\mathrm{MscCa}$, also referred to as a stretch-activated cation channel (SAC), was recognized more than 20 years ago during patch clamp studies of chick skeletal muscle, and shown to be present in most, if not all, eukaryotic cells $(8,9)$. MscCa displays a range of permeability properties indicating a heterogeneous composition. Initially, MscCa was proposed to derive its stretch sensitivity from the cytoskeleton (CSK)(8), but MscCa activity in CSK-deficient membrane vesicles and liposomes indicates the channel can also be gated by forces within the bilayer $(18,29)$. A membrane protein solubilization/reconstitution procedure similar to that used to identify MscL, a prokaryotic mechano-sensitive channel (25), was used to identify a Xenopus membrane protein fraction that reconstituted MscCa activity and was abundant in a $\sim 80 \mathrm{kDa}$ protein fraction (18). Immunological methods demonstrated TRPC1 presence in the active fraction (18). Furthermore, heterologous expression of hTRPC1 increased MscCa activity, while anti-sense 
hTRPC1 reduced endogenous/background oocyte channel activity (18). Overexpression of hTRPC1 was also reported to increase MscCa activity in Chinese hamster ovary $(\mathrm{CHO})$ cells (18).

However, several recent developments warrant a novel study and discussion. First, a TRPC1-/- knock-out mouse has been generated that shows no apparent phenotype (6). This work further demonstrates that TRPC1 is not a store-operated channel (SOC) (6). Second, mammalian cell lines can display endogenous MscCa activity similar to that associated with hTRPC1 overexpression (18), and third, a closely related TRPC family member, TRPC6, has been reported to function as MscCa (23). Additionally, TRPA1 has also been implicated in mechanosensation of the nematode C. elegans and was proposed as a candidate mechanosensor in mammalian hearing, although knock out studies in the mouse failed to confirm this hypothesis $(4,5,14)$.

In this report, we have studied the functional expression of TRPC1 and TRPC6 in both transiently transfected $\mathrm{CHO}$ and COS cells in comparison with the mechano-gated $\mathrm{K}_{2 \mathrm{P}}$ channel TREK-1 (12). Our study demonstrates that the functional expression of both TRPC1 or TRPC6 is problematic, thus leaving open the question for a specific role of these subunits in MscCa activity.

\section{Results}

\section{Expression of TRPC1 and TREK-1 in transiently transfected COS cells}

Although the results presented by Maroto and colleagues were promising (18), the reported 10-fold increase in MscCa activity seen with expression of hTRPC1 was much less than the 1000-fold increase achieved with overexpression of other channels such as the mechano-sensitive $\mathrm{K}_{2 \mathrm{P}}$ channel TREK-1 (Fig. 1a-b). Furthermore, data presented here, and representing a much larger sample than originally studied, indicate that control COS-7 can express levels of background 
MscCa activity that is as high as that reported in hTRPCl-transfected cells in CHO cells (18)(Fig. 1c-d). These channels are cationic non-selective with a conductance of $28.8 \pm 0.3 \mathrm{pS}(\mathrm{n}=11)$ (Fig. 2$)$. The mean peak current amplitude was $9.71 \pm 1.34$ $\mathrm{pA}(\mathrm{n}=70)$ and $10.23 \pm 0.99 \mathrm{pA}(\mathrm{n}=140)$ for hTRPC1 and empty pIRES2 EGFP expressing cells, respectively. At the 0.05 level, the means are not significantly different (one-way ANOVA). After removing the silent patches (with no channel activity at $-80 \mathrm{~mm} \mathrm{Hg}$ ), the mean peak current amplitude became $13.59 \pm 1.46 \mathrm{pA}$ $(\mathrm{n}=50)$ and $14.77 \pm 1.16 \mathrm{pA}(\mathrm{n}=97)$ for hTRPC1 and empty pIRES2 EGFP expressing cells, respectively. Again, at the 0.05 level, the means are not significantly different (one-way ANOVA). Moreover, the background MscCa activity is not stable and varies from cell-to-cell within the same culture and from experiment-to-experiment (Fig. 3). The basis of this variability and whether it arises through heterogeneities in endogenous TRP channel expression remains to be determined. When investigated at different pressures over a range of $80 \mathrm{~mm}$ $\mathrm{Hg}$, no significant difference was found between the TRPC1 and the empty expression vector expressing cells, unlike the TREK-1 expressing cells (20) (Fig. 4).

\section{Is TRPC1 expressed at the plasma membrane?}

An issue concerns the proportion of expressed hTRPC1 that is inserted in the plasma membrane (11). Unlike with TREK-1 (Fig. 5a) and in agreement with previous studies (11), most of the expressed hTRPC1 fails to reach the plasma membrane of mammalian cells including $\mathrm{COS}$ and $\mathrm{CHO}$ cells but instead accumulates in the endoplasmic reticulum (Fig, 5b, 5d)(Supp movies 1 and 2). TRPC1 was distributed throughout the cell and not obviously located at the cell membrane in both $\mathrm{CHO}$ and COS cells (Fig, 5b, 5d)(Supp movies 1 and 2). In comparison, hTRPC1 expressed in frog oocytes was apparently concentrated in the surface membrane (Fig. 5c). 


\section{Expression of TRPC1 in transiently transfected CHO cells}

For $\mathrm{CHO}$ cells (Fig. 6), over a period of one year, we observed a random variation of three orders of magnitude in background stretch-activated currents (Fig. 6 black squares). $\mathrm{CHO}$ cells transfected with hTRPC1 had the same basic response (Fig. 6 red circles). hTRPC1 transfected and untransfected cells are indistinguishable. The unitary conductance was $(34 \pm 2.6 \mathrm{pS})$, similar to that reported by Maroto et al. and the reversal potential was around $0 \mathrm{mV}$ (18)(not shown). The data of Maroto et al., obtained in $\mathrm{CHO}$ cells are indicated in Figure 6 as blue asterisks and they fall in the same range of current amplitude as those in non-transfected control cells observed in the present study (18). Therefore, we cannot conclude that an increase in a stretch-activated current in these mammalian cells was due to the expression of the cloned hTRPC1 channel.

\section{Expression of TRPC6 in transiently transfected COS cells}

Membrane insertion of TRPC1 can be increased by co-transfection with other TRPCs (11) suggesting that endogenous TRPCs may combine with hTRPC1 to form heteromeric channels $(11,24,26)$. As a consequence, any variation in endogenous TRPC expression with clone, passage number and/or culture condition could influence the level of expressed as well as endogenous channel activity. TRPC6 is of particular interest because recent results indicate that hTRPC6 expression in CHO cells also leads to increased MscCa activity (23). This would be consistent with the proposal that TRPC6 participates in the pressuredependent myogenic contraction of cerebral arteries (27), and indeed, antisense oligonucleotides to TRPC6 attenuate the arterial smooth muscle depolarization and constriction caused by elevated intraluminal pressure (27). However, an indirect mechanism involving diacylglycerol activation following phospholipase 
C stimulation has initially been proposed to account for the role of TRPC6 in the arterial myogenic contraction (27).

We have transiently transfected TRPC6 into COS cells and examined the amplitude of the stretch-activated current (Fig. 7). Preliminary experiments demonstrated that TRPC6 was highly expressed at the protein level upon transfection (Fig. 7a). However, the amplitude of the mechano-sensitive currents recorded in the cell-attached patch configuration was not different between the mock-transfected and the TRPC6-transfected cells (Fig. 7b-c), although intracellular OAG application (100 $\mu \mathrm{M})$ consistently increased channel activity, in agreement with previous reports (10). Again, as previously observed for TRPC1, a large variability was observed within each transfection (Fig. 7d-e).

\section{Discussion}

All together these results fail to confirm a significant role for TRPC1 or TRPC6 in stretch-activated channels when expressed in either COS or $\mathrm{CHO}$ cells $(18,23)$. However, in the same experiments, expression of the K2P channel TREK-1 yields reproducible large amplitude stretch-activated $\mathrm{K}^{+}$currents as previously described $(13,20)$.

Stretch-sensitivity of a channel in the patch does not prove the channel functions as a mechanotransducer either under physiological or pathological conditions. One also needs to show that modifying channel activity/expression can affect a mechanically-sensitive process $(3,17)$. However, TRPC6-/- mice actually show increased rather than decreased myogenic tone, and are hypertensive (7). This unexpected phenotype has been interpreted as arising from upregulation of TRPC3 that serves a similar function as TRPC6 (7). The results of

a TRPC1-/- knock out mouse are even more puzzling since this animal shows no 
phenotype, and develops normally even though TRPC1 is the most widely expressed TRPC subunit and has been implicated to be an essential component of the store-operated channel (6). Perhaps less surprising, because of the role of TRPC6, TRPC1 deletion does not affect vascular mechanotransduction, nor does it lead to any detectable upregulation of other TRPCs (6). These results may indicate a normal redundancy of TRPC channels within cells in which several channels perform similar functions (2). An analogous situation seems to apply in E.coli where knock out of MscL alone produces no phenotype, and only when MscS is also deleted, do the cells show abnormal growth in response to osmotic stress (16). Similar redundant mechanisms in vertebrate cells may compensate when specific MscCa activity is blocked during Xenopus development (28). Clearly, the situation with MscCa contrasts with that seen with the stretchactivated $\mathrm{K}^{+}$channels encoded by the $\mathrm{K}_{2 \mathrm{P}}$ channel subunits where TREK-1 expression in either Xenopus oocytes or COS-7 cells results in robust mechanosensitive $\mathrm{K}^{+}$currents in excised patches $(\geq 1 \mathrm{nA}$; Fig. 1a-b)(12, 20). Moreover, TREK-1-/- mice are more sensitive (inactivation of a stretch-activated hyperpolarizing $\mathrm{K}^{+}$channel) to mechanical stimuli indicating that eukaryotic MS channels can be studied at both the molecular and organismal level (1).

The experiments of Lauritzen et al show that expression of a channel protein can change cell structure regardless of whether or not the channel is permeant, so the proper control for transfection is far from obvious (15). Similar changes in background currents may be elicited by cytoskeletal disrupting agents. For example PC12 cells treated with cytochalasin-D increased the background MSC current 3 fold $(2.9 \pm 0.6 \mathrm{pS} / \mathrm{mmHg}[\mathrm{n}=7]$ vs $0.9 \pm 0.1 \mathrm{pS} / \mathrm{mmHg}[\mathrm{n}=6]$, data not shown). Background MscCa are often functionally concealed, but they can be exposed by repeated stimulation $(19,21,22)$. In any case, given that all cells have 
background $\mathrm{MscCa}$, the minimum requirement for dependable results should be a double blind experiment.

In conclusion, although several TRPC channel members, including TRPC1 and TRPC6, have been proposed to form MscCa in vertebrate cells $(18,23)$, the functional expression of these subunits in heterologous systems remains problematic. In particular, the variable levels of background MscCa expression seen in all mammalian cell lines, and the yet to be defined factors that regulate this expression complicate experiments comparing activity in mammalian sublines. Clearly, further studies are needed to identify the interacting and regulatory components required for the proper maturation and/or functioning of this class of ion channels. The present results are in sharp contrast with the original reports claiming that both TRPC1 and TRPC6 are components of the MscCa $(18,23)$. Differences in cell lines, transfection methods, trafficking, protein maturation, endogenous subunits may certainly be at play and explain these experimental discrepancies. However, in Xenopus oocytes, TRPC1 may reach the plasma membrane (Fig. 5d) and thus contribute to the activity of MscCa (18). 
Acknowledgements: JF, DB, AP and EH are grateful to the ANR 2005 Cardiovasculaire-obésité-diabète, to the Association for Information and Research on Genetic Kidney Disease France, to the Fondation del Duca, to the Fondation de France, to the Fondation de la Recherche Médicale, to EU Marie-Curie fellowships, to INSERM, to Human Frontier Science program and to CNRS for support. RM and $\mathrm{OH}$ thanks the DOD and NCI for support. The Buffalo group was supported by the NIH and the Oshei Foundation. We are grateful to Wade J. Sigurdson, Director of the Confocal Microscope and 3-D Imaging Facility at UB, for help with imaging. We are grateful to both Annmarie Surprenant (Manchester, UK) and David Clapham (Harvard, USA) for personal communications. 
Figure 1: Stretch-activated currents averaged across many patches in the cellattached patch configuration in transiently transfected COS-7 cells. a) Empty expression vector $(n=16)$. b) TREK-1 $(n=10)$. c) Mock transfection with the empty expression vector $(n=70)$. d) hTRPC1 $(n=70)$. The pressure pulse protocol is shown in top panel a. Each color indicates a pressure value. The holding potential was $-100 \mathrm{mV}$ for panels a-b and $0 \mathrm{mV}$ for panels c-d. Currents are inward in $a$ and $b$ and outward in $d$.

Fig. 2: a) Cell-attached patch single channel recordings at different pressures as indicated at a holding potential of $-100 \mathrm{mV}$ in a mock-transfected COS-7 cells. b) Single channel I-V curves in the presence of external $150 \mathrm{mM} \mathrm{NaCl}(\mathrm{n}=4)$, external choline chloride $(n=3)$ and external Na gluconate $(n=3)$ demonstrating that channels are nonselective and cationic. Currents were recorded in mocktransfected COS cells during a pressure pulse of $-30 \mathrm{~mm} \mathrm{Hg}$.

Fig. 3: a) shows peak current amplitude elicited by a $-80 \mathrm{~mm} \mathrm{Hg}$ pressure in the cell-attached configuration measured at $-100 \mathrm{mV}$. hTRPC1 expressing cells (shown in red; $\mathrm{n}=70$ ) and mock-transfected cells expressing the empty expression vector (pIRES2 EGFP) (shown in black; $\mathrm{n}=140$ ) are illustrated. $\mathrm{b}$ ) shows normalized frequency as a function of peak current amplitude ( $5 \mathrm{pA}$ bins). Patches from hTRPC1 expressing cells $(n=70)$ are shown by red bars while control currents $(\mathrm{n}=140)$ are shown in black.

Fig. 4: a) Pressure-response curves for COS cells transfected with the empty expression vector $(n=70)$, hTRPC1 $(n=70)$ or mTREK-1 $(n=10)$. TREK1 produces large currents unlike TRPC1. b) Expanded current scale for the mock and hTRPC1 
conditions showing that the pressure dependence of the mock and TRPC1 cells are the same. Currents were recorded in the cell-attached patch configuration at a holding potential of $-100 \mathrm{mV}$ (mock and TRPC1) or $0 \mathrm{mV}$ (TREK-1).

Figure 5: a) Confocal image of CHO cells transfected with EYFP-mTREK-1. b) Confocal image of $\mathrm{CHO}$ cells transfected with hTRPC1-EYFP. Both sections are at about the same height above the coverslip. c) A high magnification confocal fluorescent image focused on the edge of an oocyte that had been injected 3 days earlier with an mRNA construct encoding enhanced green fluorescence protein (eGFP) attached to the C-terminus of hTRPC1. GFP-TRPC1 is concentrated at the membrane surface of the Xenopus oocyte. The insert shows the same oocyte at lower magnification (1 $\mathrm{mm}$ in diameter). d) Expression of flag-tagged hTRPC1 in transiently transfected COS cells. Nuclei were stained with DAPI in blue and hTRPC1 expression is shown in red. Note the obvious localization of hTRPC1 in the endoplasmic reticulum.

Fig. 6: Pressure sensitive peak currents in $\mathrm{CHO}$ cells for patches collected over several months. Notice the log scale that suppresses the apparent scatter. Membrane potential: $-90 \mathrm{mV}$. hTRPC1 transfected $\mathrm{CHO}$ cells (red circles) and control cells (black squares). The data of Maroto et al. (18) are shown as blue asterisks for TRPC1 expressing cells and the control is shown by C.

Fig. 7: a) Expression of amino terminal HA tagged hTRPC6 in COS cells. Nuclei were stained with DAPI in blue and hTRPC6 expression is shown in red. b) Stretch-activated currents averaged across 40 patches in the cell-attached patch configuration in transiently transfected COS-7 cells with the empty expression 
vector $(n=40)$. c) hTRPC6 $(n=41)$. The pressure pulse protocol is shown in panel b. d) Effect of $100 \mu \mathrm{M}$ OAG on channel activity of an inside-out patch expressing hTRPC6. e) Pressure sensitive peak currents in COS cells for patches collected over several transfections. hTRPC6 transfected $\mathrm{CHO}$ cells (blue circles) and control cells (black circles). f) Normalized frequency as a function of peak current amplitude (5 pA bins). Patches from hTRPC6 expressing cells $(n=41)$ are shown by blue bars while control currents $(n=40)$ are shown in black. In b-f, the holding potential was $-100 \mathrm{mV}$ and currents are inward. The pressure stimulation was -60 mm Hg.

Movie Supp 1: Movie (.avi) of 3D reconstruction of EYFP labelled TREK-1 showing localization in the plasma membrane (The DivX codec is recommended: http://www.divx.com/divx/windows/author/?lang=en).

Movie Supp 2: Movie (.avi) of 3D reconstruction of EYFP labelled hTRPC1 showing a presence throughout the cell but no concentration in the plasma membrane (The DivX codec is recommended: http://www.divx.com/divx/windows/author/?lang=en). 


\section{Materials and methods}

Cell culture, plasmid construction, transfection with DEAE-dextran or Fugene, and the electrophysiological procedures have been extensively detailed elsewhere (18, 20). TREK-1 (Accession No: AY736359), hTRPC1 (alternatively spliced sequence accession $\mathrm{N}^{\circ}$ : NM003304) and hTPRPC6 (Accession $\mathrm{N}^{\circ}$ : NM 004621) were transiently transfected in CHO or COS-7 cells. The same pIRES2 EGFP expression vector was used for functional expression of both channel types in transiently transfected COS and CHO cells. We routinely used $0.5 \mu \mathrm{g}$ DNA per $35 \mathrm{~mm}$ diameter plate containing 30,000 cells. Patch pipettes were 1.5 M . Membrane stretch (ALA HSPC-1 pressure clamp) was applied as previously described (13). Routinely, the pipette solution contained (in $\mathrm{mM}$ ): $150 \mathrm{NaCl}, 5$ $\mathrm{KCl}, 3 \mathrm{MgCl}_{2}, 1 \mathrm{CaCl}_{2}$, and 10 HEPES, pH 7.4 with $\mathrm{NaOH}$ and the bath solution contained (in mM): $155 \mathrm{KCl}, 5$ EGTA, $3 \mathrm{Mg}^{2+}, 10$ HEPES at $\mathrm{pH}$ 7.2. Aminoterminal EYFP-tagged mTREK-1 channel and carboxy terminal EYFP-tagged hTRPC1 were used for channel localization in transfected mammalian cells. The EYFP-mTREK-1 fusion protein showed no functional difference with the WT channel. In some experiments an amino terminal Flag tagged hTRPC1 construct and an amino terminal HA tagged hTRPC6 were used. After mounting, specimens were observed using an epifluorescence microscope (Axioplan 2, Carl Zeiss) with appropriate filters. Images were recorded with a cooled CCD camera (Coolsnap HQ, Photometrics) driven by Metavue software. Three dimensional reconstructions and stereo pairs were made using a ZEISS confocal microscope and relevant software. Other methods are essentially as described by Maroto et $\mathrm{al}^{1}$. CHO-K1 cells (ATCC, Manassa, VA) were cultured in RPMI 1640 medium 
supplemented with $25 \mathrm{mM}$ Hepes and glutamine, $8 \%$ fetal calf serum, $1 \mathrm{mM} \mathrm{Na}$ pyruvate, $4.5 \mathrm{~g} / \mathrm{L}$ glucose and antibiotics at $37^{\circ} \mathrm{C}$ in a humidified $95 \% \mathrm{O}_{2}-5 \% \mathrm{CO}_{2}$ atmosphere. Cells were transfected with TRPC1-XOOM using Fugene HD (Roche, Indianapolis, IN) and sorted by FACS. Patch-clamp experiments were carried out after 2-5 days of seeding cells on glass cover slips. Xenopus oocytes were injected with mRNA transcripts of eGFP-hTRPC1 and cells were studies 3-4 days after injection. 


\section{References}

1. Alloui A, Zimmermann K, Mamet J, Duprat F, Noel J, Chemin J, Guy N, Blondeau N, Voilley N, Rubat-Coudert C, Borsotto M, Romey G, Heurteaux C, Reeh $P$, Eschalier A, Lazdunski M (2006) TREK-1, a K+ channel involved in polymodal pain perception. EMBO J 25:2368-2376

2. Beech DJ (2005) TRPC1: store-operated channel and more. Pflugers Arch 451:53-60

3. Bode F, Sachs F, Franz MR (2001) Tarantula peptide inhibits atrial fibrillation. Nature 409:35-36

4. Corey DP (2006) What is the hair cell transduction channel? J Physiol 576:2328

5. Corey DP, Garcia-Anoveros J, Holt JR, Kwan KY, Lin SY, Vollrath MA, Amalfitano A, Cheung EL, Derfler BH, Duggan A, Geleoc GS, Gray PA, Hoffman MP, Rehm HL, Tamasauskas D, Zhang DS (2004) TRPA1 is a candidate for the mechanosensitive transduction channel of vertebrate hair cells. Nature 432:723-730 6. Dietrich A, Kalwa H, Storch U, Mederos YSM, Salanova B, Pinkenburg O, Dubrovska G, Essin K, Gollasch M, Birnbaumer L, Gudermann T (2007) Pressureinduced and store-operated cation influx in vascular smooth muscle cells is independent of TRPC1. Pflugers Arch

7. Dietrich A, Mederos YSM, Gollasch M, Gross V, Storch U, Dubrovska G, Obst M, Yildirim E, Salanova B, Kalwa H, Essin K, Pinkenburg O, Luft FC, Gudermann T, Birnbaumer $L$ (2005) Increased vascular smooth muscle contractility in TRPC6-/- mice. Mol Cell Biol 25:6980-6989

8. Guharay F, Sachs F (1984) Stretch-activated single ion channel currents in tissue-cultured embryonic chick skeletal muscle. J Physiol 352:685-701

9. Hamill OP (2006) Twenty odd years of stretch-sensitive channels. Pflugers Arch 453:333-351

10. Hofmann T, Obukhov AG, Schaefer M, Harteneck C, Gudermann T, Schultz G (1999) Direct activation of human TRPC6 and TRPC3 channels by diacylglycerol. Nature 397:259-263

11. Hofmann T, Schaefer M, Schultz G, Gudermann T (2002) Subunit composition of mammalian transient receptor potential channels in living cells. Proc Natl Acad Sci U S A 99:7461-7466

12. Honoré E (2007) The neuronal background K2P channels: focus on TREK-1. Nature reviews neuroscience 8:251-261

13. Honoré E, Patel AJ, Chemin J, Suchyna T, Sachs F (2006) Desensitization of mechano-gated K2P channels. Proc Natl Acad Sci U S A 103:6859-6864

14. Kindt KS, Viswanath V, Macpherson L, Quast K, Hu H, Patapoutian A, Schafer WR (2007) Caenorhabditis elegans TRPA-1 functions in mechanosensation. Nat Neurosci 10:568-577 
15. Lauritzen I, Chemin J, Honoré E, Jodar M, Guy N, Lazdunski M, Jane Patel A (2005) Cross-talk between the mechano-gated K2P channel TREK-1 and the actin cytoskeleton. EMBO Rep 6:642-648

16. Levina N, Totemeyer S, Stokes NR, Louis P, Jones MA, Booth IR (1999)

Protection of Escherichia coli cells against extreme turgor by activation of MscS and MscL mechanosensitive channels: identification of genes required for MscS activity. Embo J 18:1730-1737

17. Maroto R, Hamill OP (2007) MscCa regulation of tumor cell migration and metastasis. Curr Tops Membr 59:485-509

18. Maroto R, Raso A, Wood TG, Kurosky A, Martinac B, Hamill OP (2005) TRPC1 forms the stretch-activated cation channel in vertebrate cells. Nat Cell Biol 7:179-185

19. Morris CE (1990) Mechanosensitive ion channels. J Membr Biol 113:93-107

20. Patel AJ, Honoré E, Maingret F, Lesage F, Fink M, Duprat F, Lazdunski M (1998) A mammalian two pore domain mechano-gated S-like $\mathrm{K}^{+}$channel. EMBO J 17:4283-4290

21. Sachs F (1997) Mechanical transduction by ion channels: how forces reach the channel. Soc Gen Physiol Ser 52:209-218

22. Sachs F, Morris CE (1998) Mechanosensitive ion channels in nonspecialized cells. Rev Physiol Biochem Pharmacol 132:1-77

23. Spassova MA, Hewavitharana T, Xu W, Soboloff J, Gill DL (2006) A common mechanism underlies stretch activation and receptor activation of TRPC6 channels. Proc Natl Acad Sci U S A

24. Strubing C, Krapivinsky G, Krapivinsky L, Clapham DE (2001) TRPC1 and TRPC5 form a novel cation channel in mammalian brain. Neuron 29:645-655

25. Sukharev SI, Blount P, Martinac B, Blattner FR, Kung C (1994) A largeconductance mechanosensitive channel in $E$. coli encoded by mscL alone. Nature 368:265-268

26. Villereal ML (2006) Mechanism and functional significance of TRPC channel multimerization. Semin Cell Dev Biol 17:618-629

27. Welsh DG, Morielli AD, Nelson MT, Brayden JE (2002) Transient receptor potential channels regulate myogenic tone of resistance arteries. Circ Res 90:248-250 28. Wilkinson NC, Gao F, Hamill OP (1998) Effects of mechano-gated cation channel blockers on Xenopus oocyte growth and development. J Membr Biol 165:161-174

29. Zhang Y, Gao F, Popov VL, Wen JW, Hamill OP (2000) Mechanically gated channel activity in cytoskeleton-deficient plasma membrane blebs and vesicles from Xenopus oocytes. J Physiol 523 Pt 1:117-130 

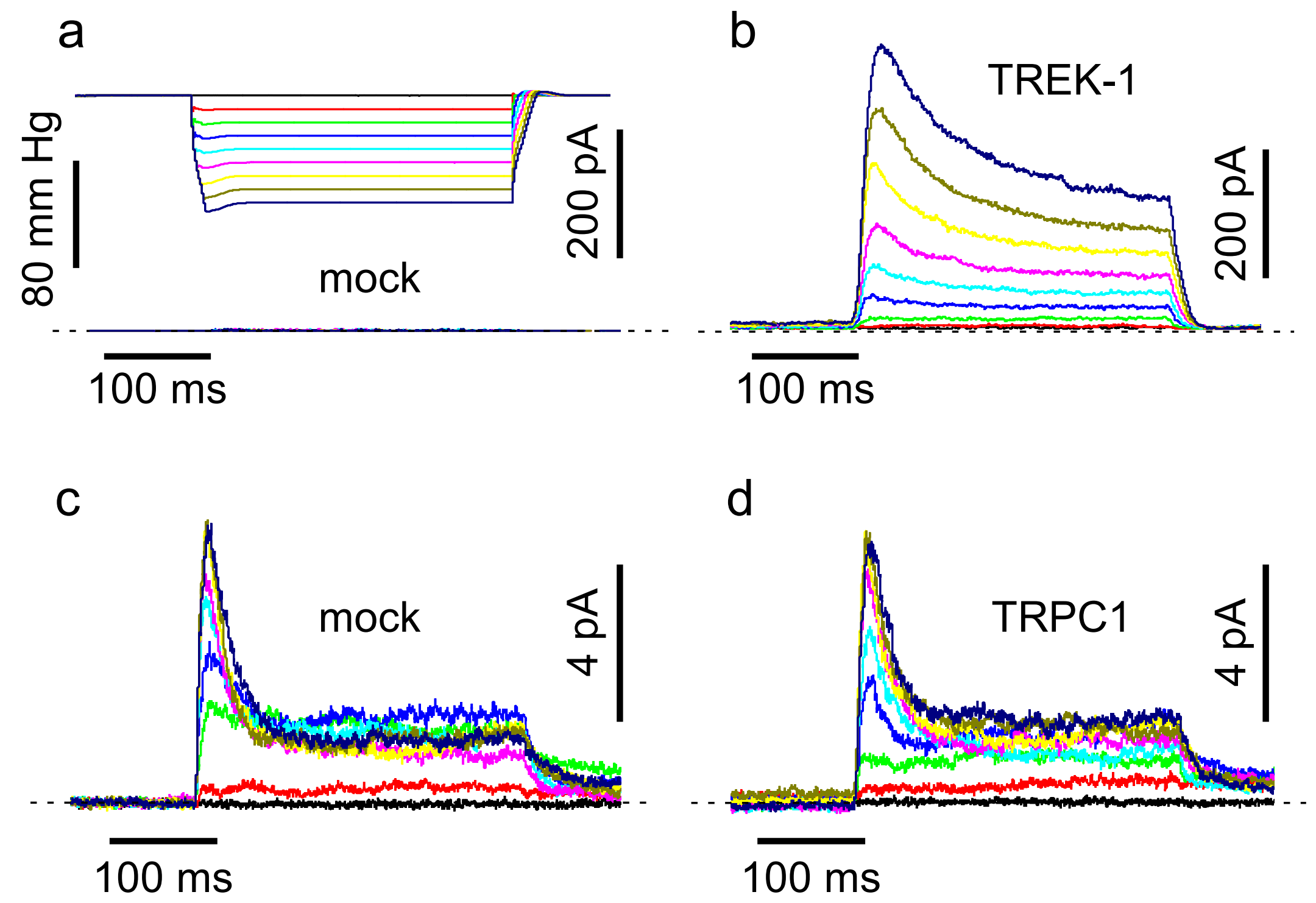

Fig. 1 
a

\section{cos cell}

Cell-attached configuration @ -100 mV

-
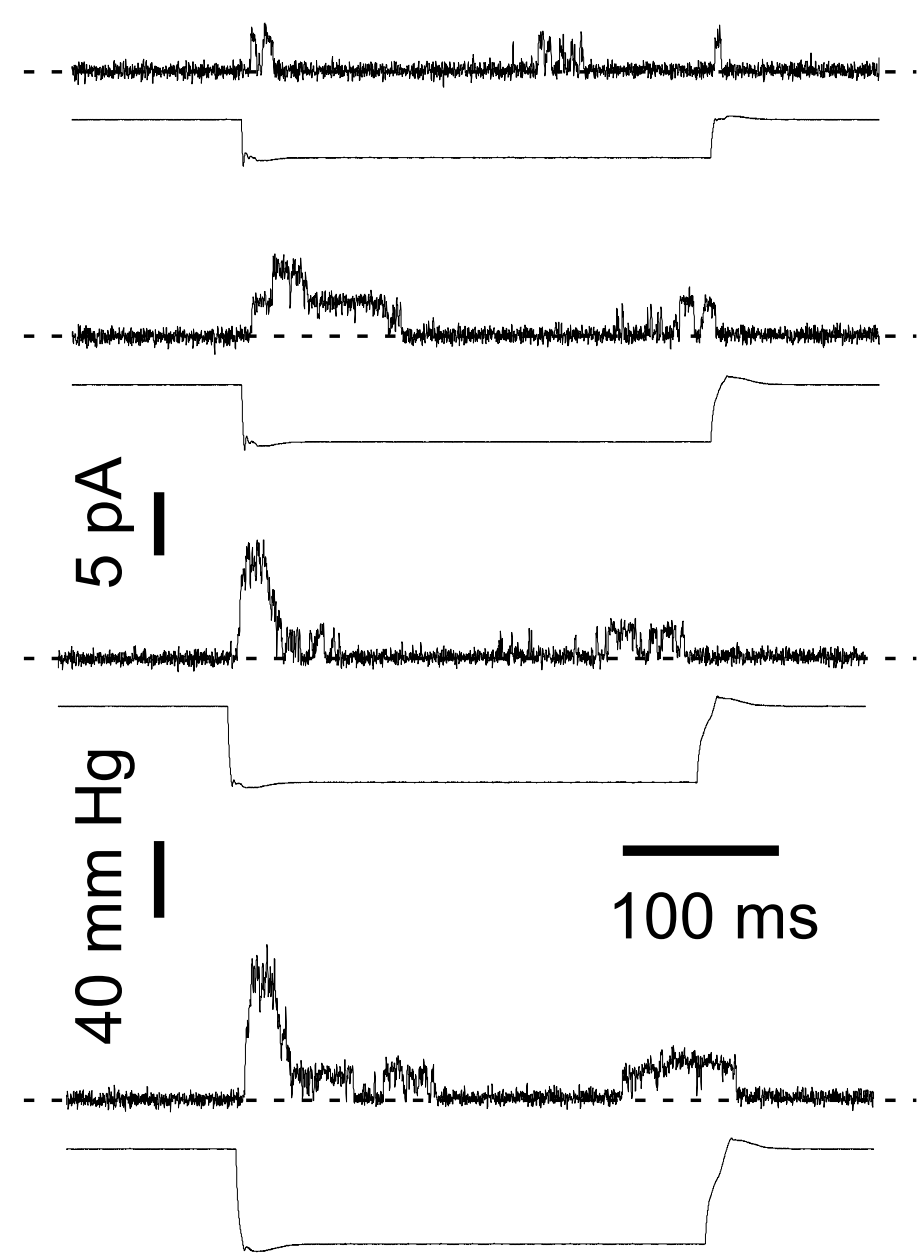

b

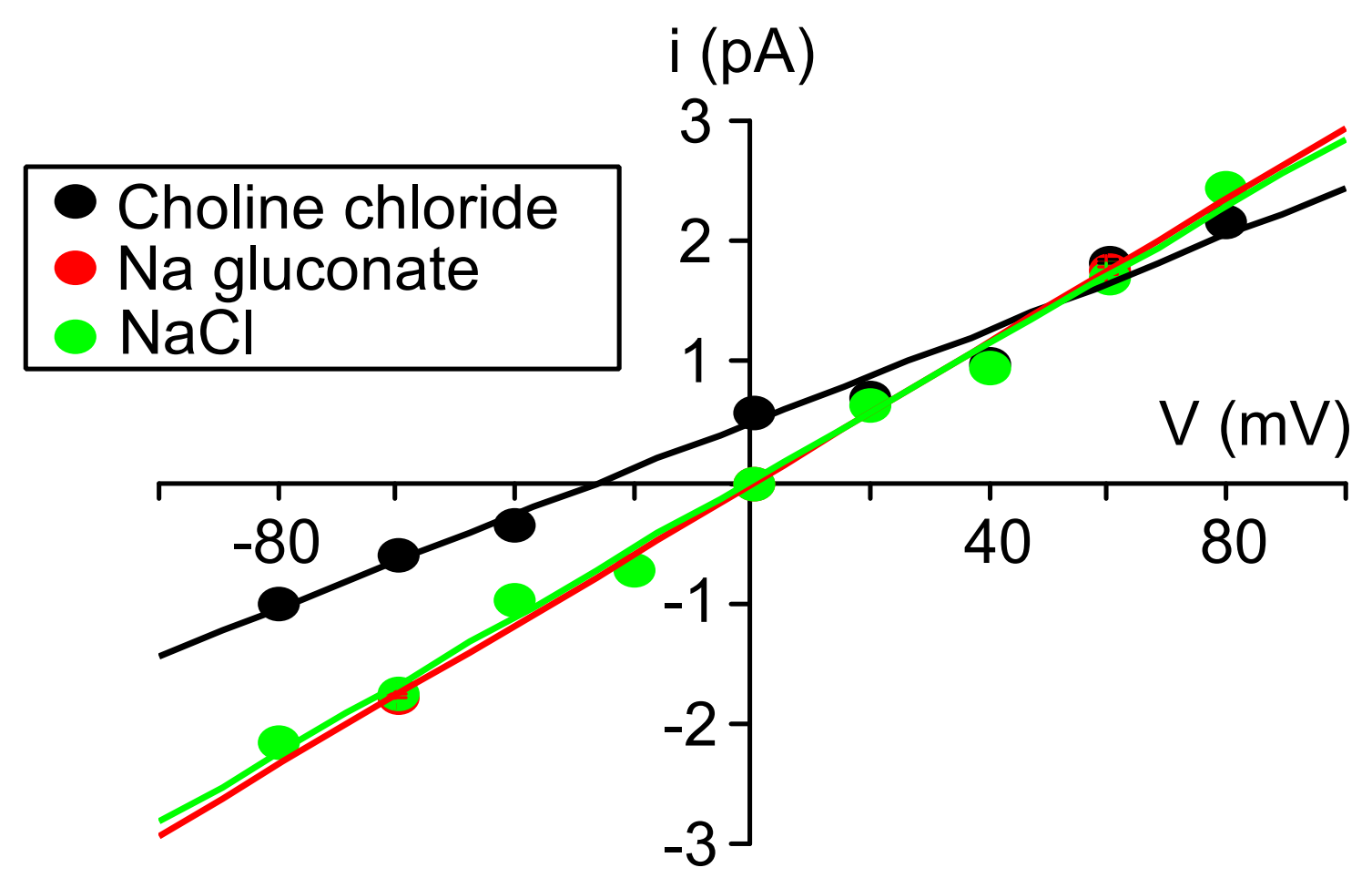

Fig. 2 

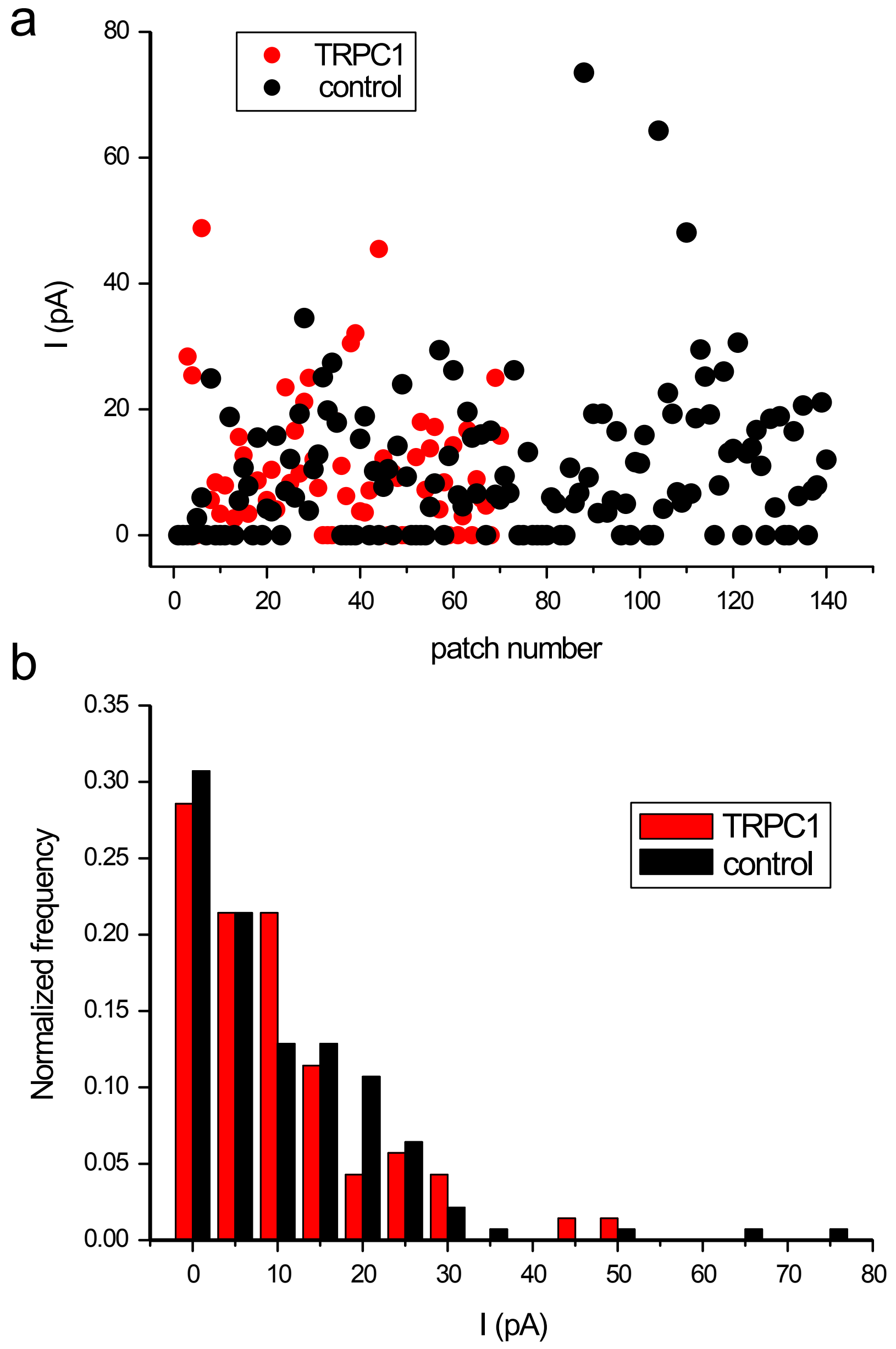

Fig. 3 

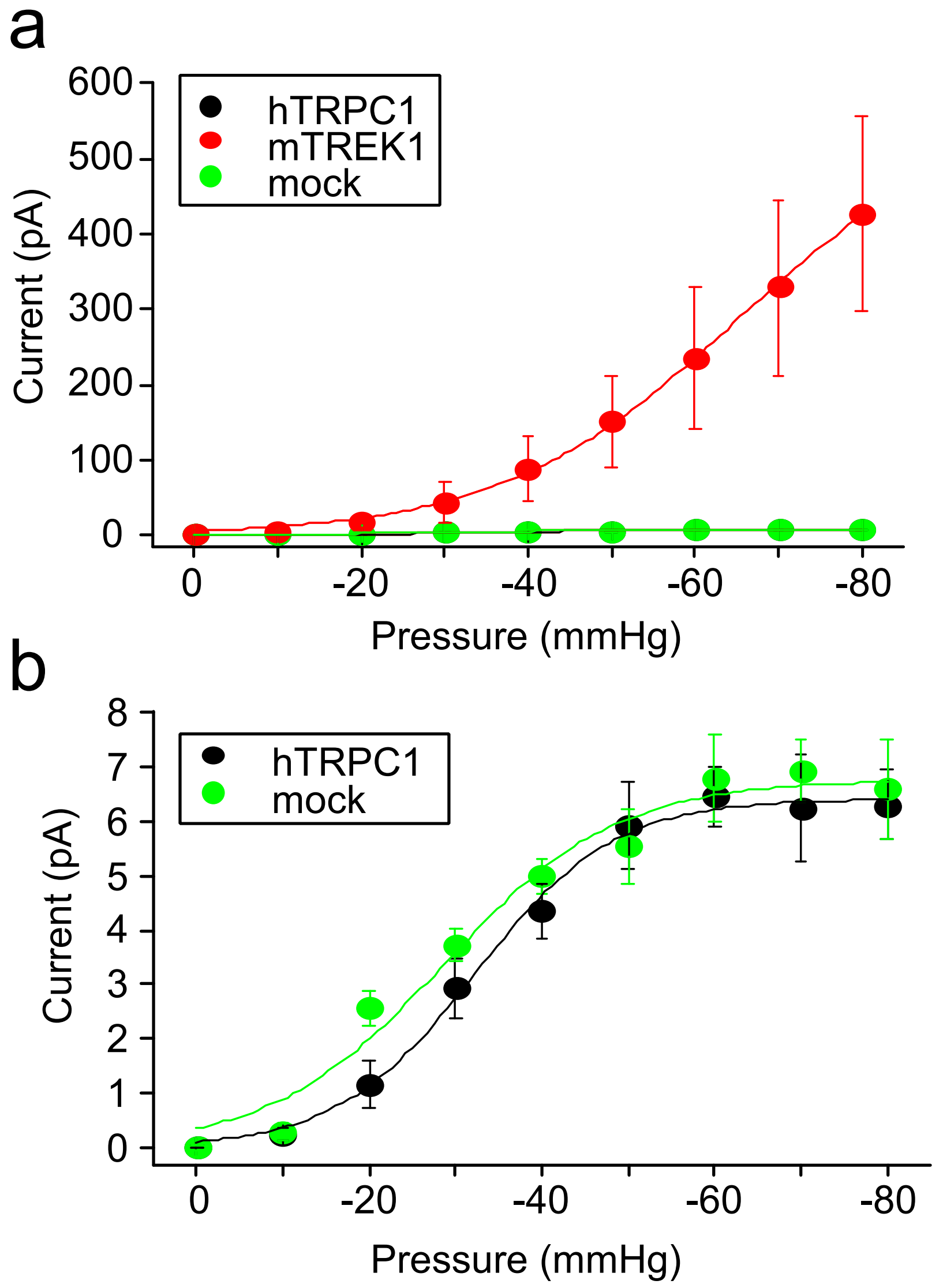

Fig. 4 

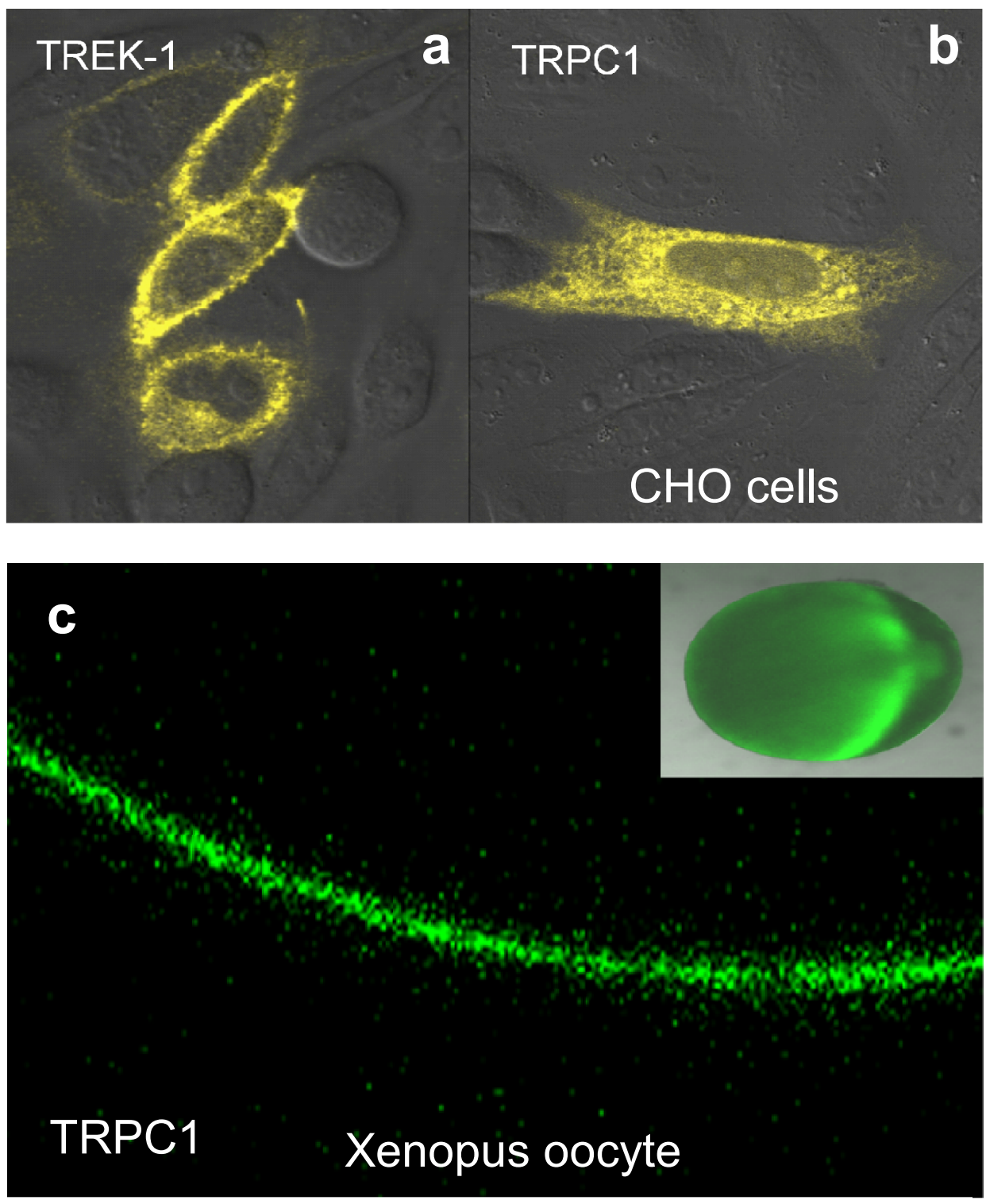

d TRPC1

COS cells

Fig. 5 


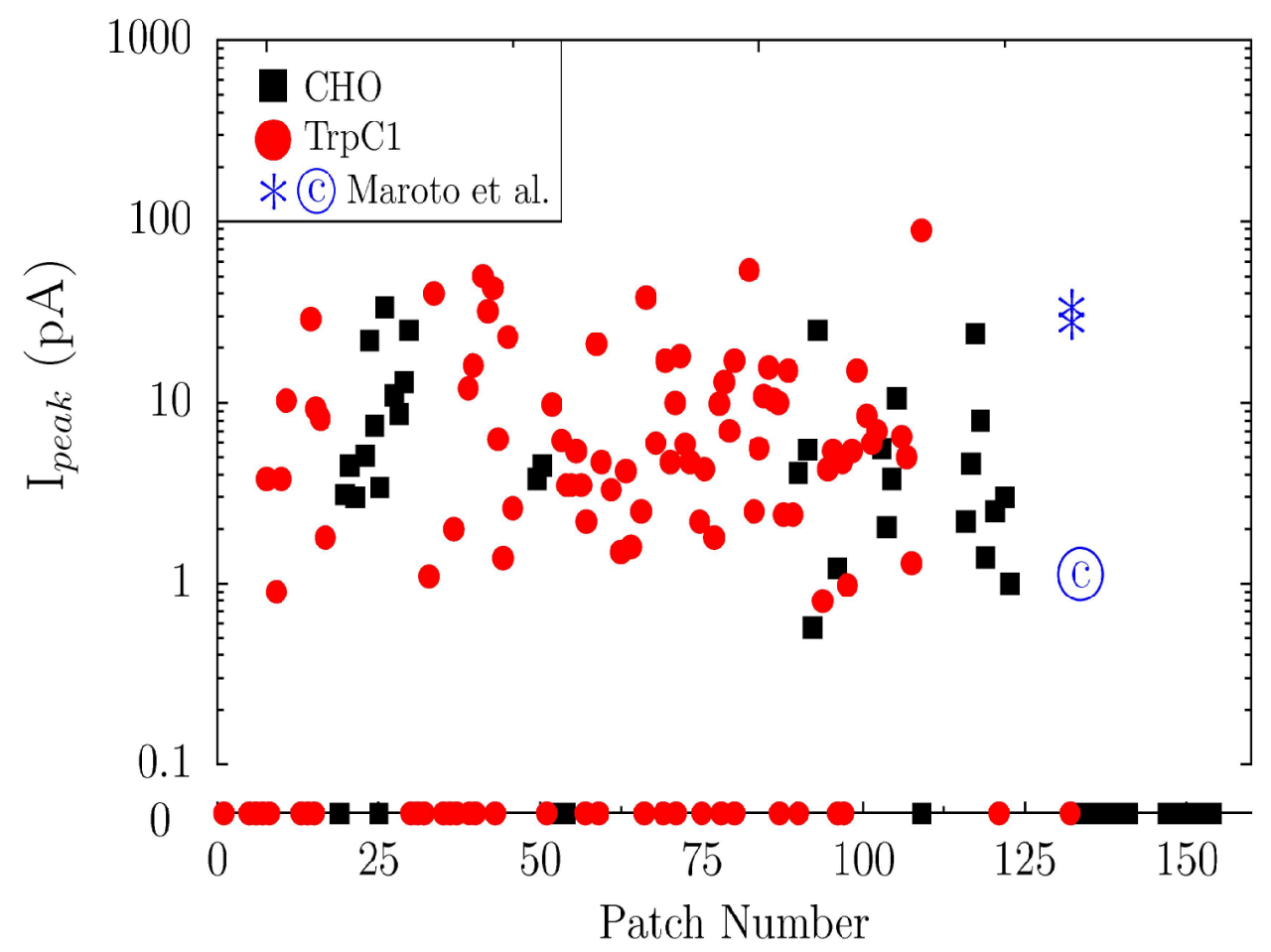

Fig. 6 


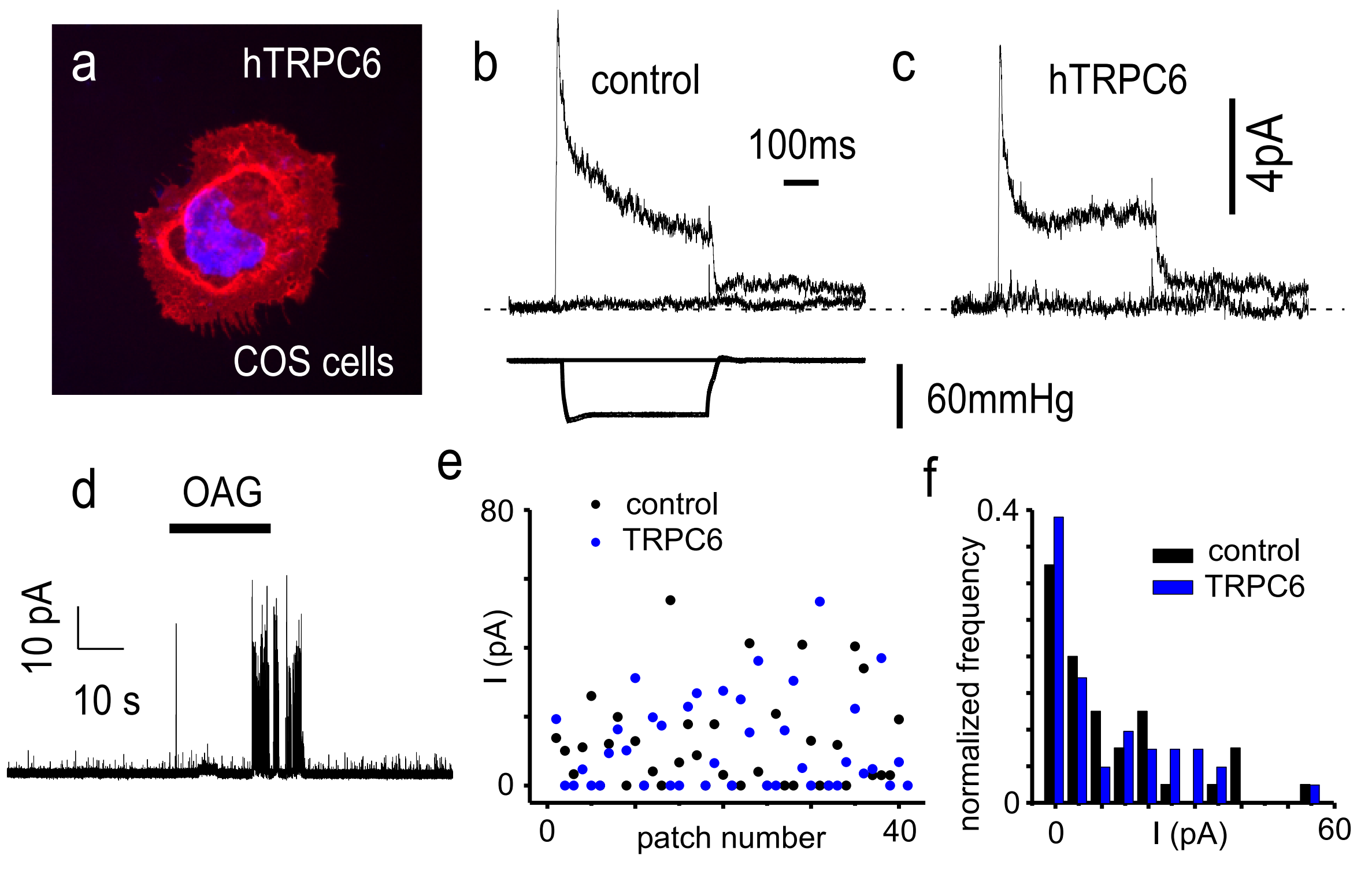

Fig. 7 